\title{
Tethered Satellites for Near and Far Earth Studies
}

\section{F. Mariani, Rome}

(Dipto. di Fisica, II Università degli Studi di Roma)
Early in the 1970's the first technically sound proposal for interconnecting space probes at different altitudes was put forward by Grossi and Colombo et al. [1]. The idea was to join a satellite to a larger orbiting mass (notably the Space Shuttle) by a tether, so that it was retained either above or below in a relatively fixed position. A number of scientific and technical applications were put forward and since then, many more have been suggested. A list can be found in the literature.

The first real mission in space to confirm the predictions on dynamic and electrodynamic behaviour, is now in the final stage of preparation. It will be a Shuttle flight in February 1991. The second, still to be approved, is planned

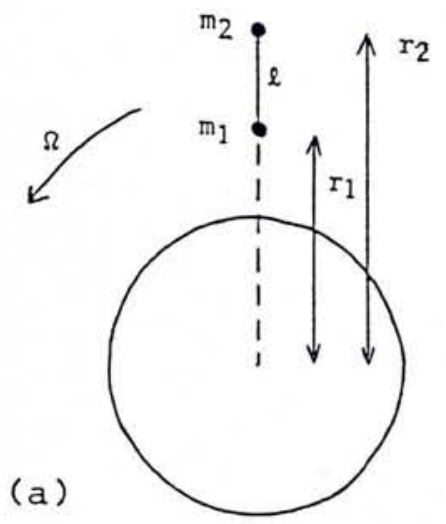

(b)

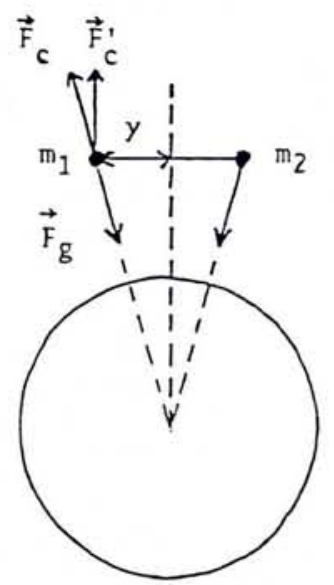

Fig. 1 - (a) Vertically stabilised system, (b) Out of plane displacement. for two or three years later and to operate at the unprecedented low altitude of $120 \mathrm{~km}$.

\section{Tether Dynamics}

A satellite orbiting around the Earth is subject to the gravitational force $F_{\mathrm{g}}=$ $-G M m / r^{2}$, where $G M=3.98601^{9} \times$ $10^{14} \mathrm{Nm}^{2} \mathrm{~kg}^{-1}$ is the Earth's gravitational constant, $m$ is the satellite mass and $r$ the geocentric distance. The centrifugal force $F_{\mathrm{c}}=m \Omega^{2} r$, where $\Omega$ is the angular velocity that in the simple case of a circular orbit, is given by:

$$
\Omega=\left[G M / r^{3}\right]^{1 / 2} \text {. }
$$

Let us consider now [6] the more complicated situation where two masses $m_{1}$ and $m_{2}$, orbiting around the Earth, are connected to each other by a tether, maintaining a rigid radial alignment as in Fig. 1a. It is evident that the gravitational acceleration $g$ on the lower mass is higher than on the upper mass, while the opposite is true for the centrifugal acceleration. Within the system, a third force is acting on each mass, i.e. the tension $T$ exerted by the tether upwards on $m_{1}$ and downwards on $m_{2}$. The system's equilibrium angular velocity is attained when the external forces sum to zero, i.e. assuming the mass of the tether to be negligible:

$$
\begin{array}{r}
\Omega=\left[G M\left(m_{1} / r_{1}^{2}+m_{2} / r_{2}^{2}\right) /\right. \\
\left.\left(m_{1} r_{1}+m_{2} r_{2}\right)\right]^{1 / 2}
\end{array}
$$

In general, along the tether at a distance $r_{0}$ there exists a point where gravitational and centrifugal forces are equal and opposite. This will coincide with the centre of gravity only if the distance separating $m_{1}$ and $m_{2}$ is very small because of the non-linear dependence of the forces upon $r$. The actual vertical force acting on $m_{1}$ and $m_{2}$ can be derived in a local reference system having its origin in $r_{0}$, a vertical $z$ axis, and an $x$ axis oriented along the flight direction (and, consequently, a $y$ axis perpendicular to $x$ and $z$ ). If a mass $m$ is located at a distance $z=r-r_{0}$ from $r_{0}$ it can easily be shown that the $z$ component of the gravity gradient force is:

$$
F_{z}=3 m \Omega^{2} z
$$

In the case of 'horizontal' alignment across the flight path, Fig. 1b, the force

$$
F_{y}=-m \Omega^{2} y
$$

whereas aligned along $x, F_{x}=0$.

If the tether is oriented in an arbitrary direction, all three force components have to be taken into account and for small displacements $x$ and $y$ from the vertical, the equations of motion in the orbital plane and perpendicular to it become

$$
\begin{aligned}
& \ddot{\theta}+3 \Omega^{2} \theta=0 \\
& \ddot{\phi}+4 \Omega^{2} \phi=0
\end{aligned}
$$

where the angles $\theta$ and $\phi$ are the corresponding (small) angular elongations $x / l$ and $y / l$. Then each mass oscillates in the two directions at angular frequencies $\sqrt{ } 3 \Omega$ and $2 \Omega$ respectively.

But now, because of the motion, an additional velocity-dependent force comes into effect, which may be positive or negative depending upon the sign of $\dot{x} / r$. The dynamics of the system becomes complicated further if retrieval and deployment are to be considered: Let us consider as an example tether retrieval; it can be shown that the tether length $I(t)$ must satisfy the equation:

$2 m \Omega l+3 m \Omega^{2} I \sin \theta \cos \theta=0$

which in the case of constant angle $\theta$ implies that $I(t)=l \exp (-a t)$ with $a=$ $(3 \Omega \cos \theta \sin \theta) / 2$. This means that the retrieval velocity must be proportional to the instantaneous length $l$.

Without going further, let us just note that the rigorous equations of motion for the most general case are very intricate, because also the mass distribution of the tether cannot be neglected, especially if it is long. It is evident that the tether itself can undergo oscillations, either longitudinal or transversal. Moreover, thermal forces due to different temperatures along the orbit and/or at different altitudes, atmospheric drag, as well as, in the case of a conductive tether, electromagnetic drag via the Lorentz force may become important.

We have to conclude that in practice the behaviour of the system is overwhelmingly complicated and so must be checked in real flight conditions. This is one of the principal aims of the first demonstration flight, which is an elec- 


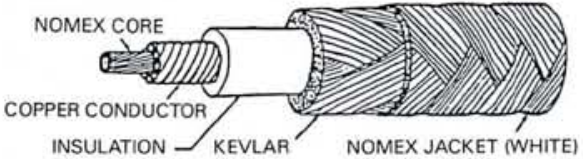

Fig. 2 - Cross-section of TSS-1 conducting tether [8].

trodynamic mission (TSS-1) [8] with a conductive tether. Table 1 gives some of the details and in Fig. 2 is depicted the cross-section of the tether, while Fig. 3 shows the shuttle prior to satellite deployment.

\section{Electrodynamic Tether}

If a conductor moves across a static magnetic field $\mathbf{B}$ an electric field $\mathbf{E}$ is induced according to the law

$$
\mathbf{E}=-\mathbf{V} \times \mathbf{B} \text {. }
$$

In the specific case of the Earth, the magnetic field embedded in the ionosphere is stationary with respect to an observer on the surface and as a consequence, a potential difference is generated between the two ends of a moving tether whose value depends upon the field intensity, the velocity and the angle of the two vectors. For a current to flow in the tether, it is necessary that space charge collected at one end of the system is dispersed along the tether (if not insulated) or at the other end (Fig. 4a). The current intensity will depend very much upon the size and shape of the two end masses, the tether resistivity and the insulation from the environment, as well as the ionospheric ion densities. For an eastward motion of the system, the electric field is oriented downwards so the upper mass is at a positive potential with respect to the lower and will collect electrons from the ionospheric environment. For a $100 \mathrm{~km}$ long tether moving at a velocity of 8 $\mathrm{km} / \mathrm{s}$ the potential difference may attain some tens of $\mathrm{kV}$. It appears that when current flows, electric power is genera-

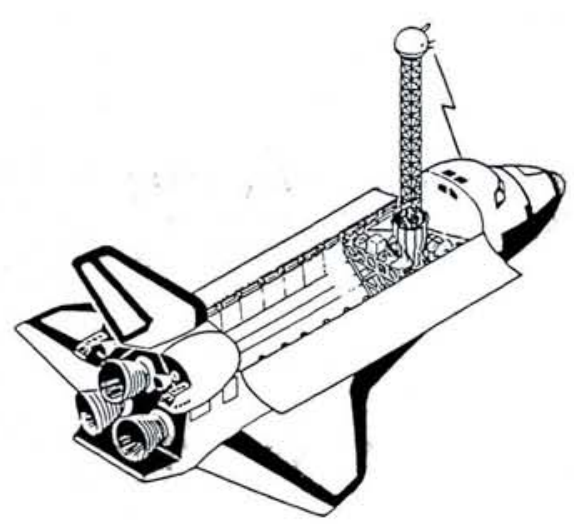

Fig. 3 - Satellite at end of extended boom ready for separation.
Table 1 - TSS-1 Tether Characteristics

\begin{tabular}{|lr|}
\hline Diameter & $2.54 \mathrm{~mm}$ \\
Deployed length & $20 \mathrm{~km}$ \\
Breaking strength & $1780 \mathrm{~N}$ \\
Design tension & $700 \mathrm{~N}$ \\
Expected load & $53 \mathrm{~N}$ \\
Maximum mass & $8.2 \mathrm{~kg} / \mathrm{km}$ \\
Maximum current & $1 \mathrm{~A}$ \\
Resistance & $0.2 \Omega / \mathrm{km}$ \\
Maximum operating voltage $5000 \mathrm{~V}$ \\
\hline
\end{tabular}

ted along the tether; power is also radiated because of moving perturbations produced in the two regions where the interaction with the ionised environment occurs. As a consequence, hydromagnetic waves can be generated and the acceleration of charged particles is also expected. The energy to excite all these phenomena is obviously derived at the expense of the kinetic energy of the system.

In this first electrodynamic mission, the conducting tether will be insulated with respect to the surrounding environment, so that the tether current will enter and leave via the conducting surfaces at the two ends. The current intensity can be varied in a number of ways: altering the ohmic resistance of the tether or the conductivity close to the collector (especially the electron collector) by, for example, plasma contactors.

A number of active experiments can be envisaged to study the properties of the plasma surrounding the system. Due to the large size of the electric circuits along which the current from the tether flows to the environment and then closes again at the other end, it is possible to study either local or even global charasteristics of the plasma far from the tether. On the other hand, since the whole system is in motion, a wake is continuously left behind which then propagates in a variety of ways through the ionosphere. It is expected that very low frequency waves will be generated with an intensity sufficient to be detected at the Earth's surface.

The electrodynamic tether system is a very special configuration of a conduc-

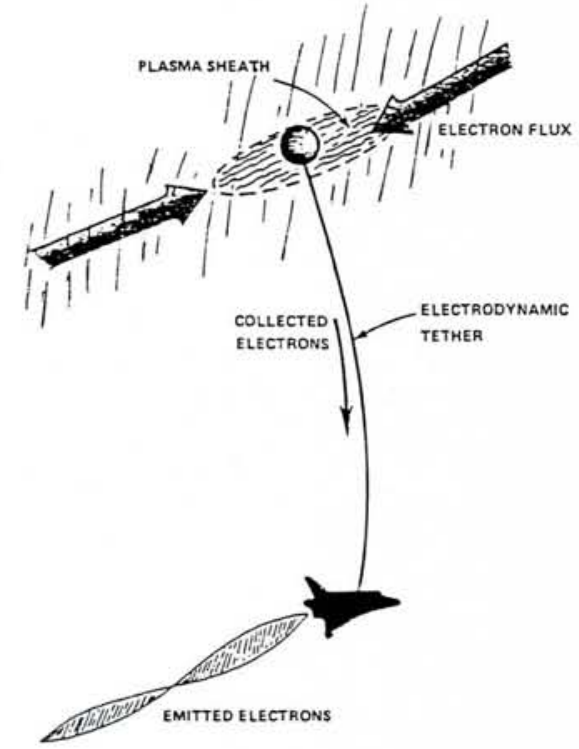

Fig. 4 - Basic components of electrodynamic experiments with an insulated conducting tether [2].

ting body in motion in a magnetic plasma (or magnetoplasma) and is a suitable instrument for studying plasma physics in general, as well as processes occurring in other environments. As an example, we can mention the case of Jupiter's satellite lo whose particular behaviour has been attributed to its electromagnetic interaction, as a conductive body, with the rapidly rotating Jovian magnetosphere.

\section{Low Altitude Atmospheric Mission}

Low altitude tethered satellites represent a unique tool for investigating the range of altitudes below $200 \mathrm{~km}$ down to $80-120 \mathrm{~km}$, for as long as several days or even weeks (Fig. 5). Whereas free satellites would rapidly lose height as friction slowed them down, the more massive mothercraft in a higher orbit serves to hold them up. Direct measurements of atmospheric properties are wanted in a number of different fields.

\section{The neutral atmosphere}

The gaseous environment of the Earth and its expansion towards the interplanetary medium, in the presence of the
Fig. 5 - Range of aerodynamic research facility.

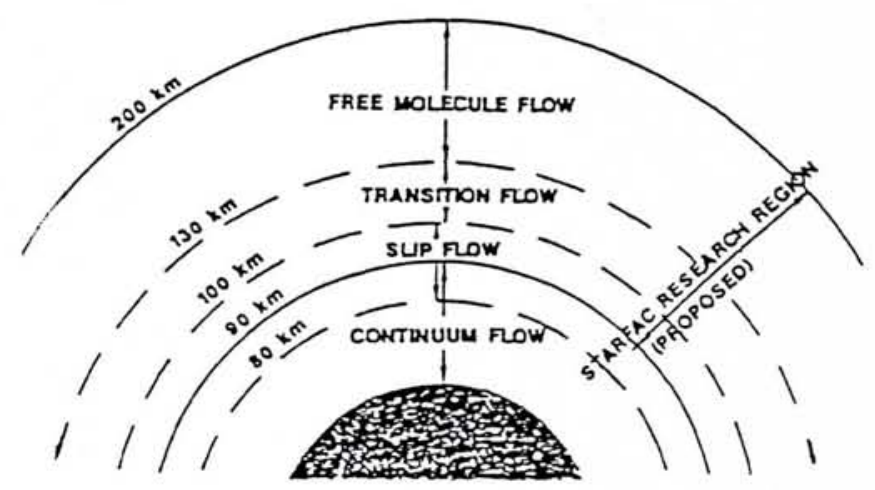


geomagnetic field, is very complex and the existing observational information is poor. Only occasional measurements have been made by rockets or, at the upper end of the altitude range, by a few satellites at perigee or during their decay phase. At an altitude of about $100 \mathrm{~km}$, the mean free path of the atmospheric gas is comparable to the typical satellite size. Above, molecular diffusion becomes predominant, i.e. the atmospheric constituents begin to separate from each other, and atomic oxygen becomes important. The turbulence typical of altitudes up to $100 \mathrm{~km}$ is no longer a dominant factor.

Among the principal scientific objectives for low altitude satellites, is the determination of the chemical composition of the neutral atmosphere, in the region where the vertical transport processes are important for the exchange of hydrogen (upwards) and nitrogen compounds (downwards). A number of different atomic and molecular species are present $\left(\mathrm{H}, \mathrm{H}_{2}, \mathrm{O}, \mathrm{O}_{2}, \mathrm{~N}_{2}\right.$, as well as traces of minor components, for example sulphur); these particles can combine with each other in a variety of ways, so that a large number of components exist, with a large spectrum of properties and lifetimes. Realistic modelling of the atmospheric composition and vertical distribution are in dire need of insitu mass spectrometric measurements. Also, chemical tracers inside large volumes and areas can be seeded in order to study the complex pattern of neutral atmospheric circulation.

Another kind of basic problem has to do with fluid dynamics: in particular, aerodynamic and heat transfer coefficients within a variety of conditions which cannot be obtained by current wind tunnel technology, because of the impossibility of making thermo-fluid-dynamic measurements in regimes where low Reynolds numbers are combined with high Mach numbers. Low altitude tethered satellites may also be used as a sort of "open wind tunnel", operating over time ranges much longer than any existing or proposed ground facility.

Also in the field of remote sensing, tethered satellites will prove invaluable for increasing the accuracy of real time cartographic maps from space.

\section{The ionized atmosphere}

In the same altitude range, the atmospheric components are subject to the ionizing action of ultraviolet solar radiation. The energy released in the process heats the gas to temperatures that increase as the altitude increases: electrons, positive ions and neutrals exhibit

\section{Physics Education Counsellors for China}

The European Physical Society has been invited by the Citizen Ambassador Program based in the USA to submit the name(s) of a leader to head an international delegation of professionals in physics education to China. The leader would be expected to participate in the development phase of the project that has been initiated by the Physical Society of the People's Republic of China. Responsibilities would include agreeing on the area of professional interest and selecting the delegation members. Expenses would be covered by the Program. No dates have yet been fixed for the delegation's visit, but a period in the early Autumn of this year or Spring of 1990 is projected. Qualified physicists interested in this project should contact the EPS Secretariat in Geneva for further details.

different vertical profiles of temperature, because thermal exchange decreases with altitude owing to the fast transition from a regime of high collisional rate between neutrals and ions to one of vanishing rate. At lower altitudes, the ions are carried along by neutral winds, while a few tens of $\mathrm{km}$ above, electric field drift motions dominate. Global motions of the ionized components embedded in the neutral gas give rise in the presence of the geomagnetic field to electrical currents (the so-called dynamo currents), which are the main source of geomagnetic variations on the ground, either regular (in particular diurnal) or irregular, as well as of Joule heating. These currents, which are essentially ionospheric at low and middle latitudes, are indeed the low latitude aspect of the complicated current circulation pattern taking place at higher latitudes. In the polar caps, owing to the high conductivity along the nearly vertical geomagnetic field lines, a system of aligned currents, called Birkeland currents, builds up connecting the lower atmosphere and ionosphere to the external magnetospheric regions, where the impinging solar wind delivers energy to the magnetosphere. This current pattern at high latitudes and in the polar caps is subject to large perturbations, in particular during geomagnetic storms and in the not infrequent event of bombardment by high energy protons from the Sun.

\section{Physica
Scripta}

An international journal for experimental and theoretical physics

Vol. T25 1989

\section{Proceedings of the 8th General Conference of the EPS Condensed Matter Division}

\section{Budapest, 6-9 A pril 1988}

Edited by: F. Beleznay, J. Kollár, I. Kovács, N. Kroó, N. Menyhárd

In addition to plenary lectures and all the individual invited talks the editors have selected contributions to the Symposia under the headings:

$\mathrm{X}$-ray and electron microscopic structural analysis; Charge density waves in solids; Localization; High $\mathrm{T}_{\mathrm{c}}$ superconductivity, Liquid crystals, Quantum tunnelling in complex systems; Transition metals in semiconductors; Spin glass models and memory; Electron spectroscopy; Novel physics of quantum wells and superlattices; Light scattering; Topics in magnetism; Chaos, fractals, turbulence; Applications; Large scale computation; Hydrogen and bonding; Liquids; Surface physics.

376 pages in A4 format covering almost 70 talks.

Orders to Physics Scripta at the address shown below; Price: SKR 900.- or \$US 160.- 


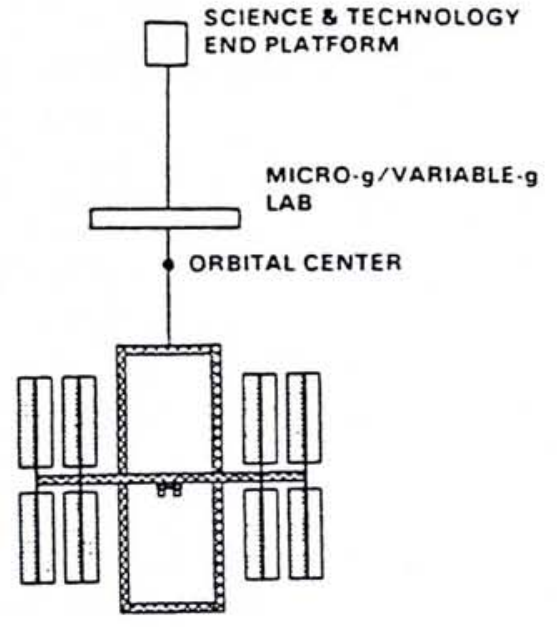

Fig. 6 - Multi-purpose experimental system with movable platform to control $\mathrm{g}$.

Below the ionospheric $F$ region, a variety of structures are to be studied, together with their temporal evolution at different scales.

\section{Geomagnetic and gravity surveys}

A definite possibility exists of using tethered satellites as platforms for making systematic high sensitivity surveys of geomagnetic and gravity anomalies. Satellite orbits are determined precisely by the actual gravity force field: the higher the orbit, the less the contribution from small scale features at ground or underground level. Information on small scale features requires the use of very low orbit satellites equipped with sensitive gradiometers: tethered satellites might be the right approach.

In-situ precise measurements of the geomagnetic field structure in the lower atmosphere, in addition to giving enlightenment on the characteristics of the ionospheric current system, will help to describe the field gradient, and thus to make fast and complete surveys of large and, above all, small scale features of internal sources. A similar improvement of knowledge is possible for the gravity field by using gravity gradiometers. Exact knowledge of the gravity field has fundamental importance in many areas. Among the various applications, solid Earth and ocean physics, Earth resources, satellite dynamical behaviour, inertial navigation can be mentioned. In particular, gravity anomalies associated with the motion of tectonic plates should be observable. Most large scale geophysical phenomena occurring at the surface of the Earth: earthquakes, volcanic phenomena, mountain build-up are in some way associated with anomalies of the density distribution below the surface down to several hundred $\mathrm{km}$, where they have their roots.

\section{Space Challenge to European Students}

To celebrate its silver Jubilee and to mark the launch of its two astronomical satellites, HST and Hipparcos, the European Space Agency (ESA) has organised an essay competition open to European students aged between 16 and 21. The main theme is "Astronomy from Space" and may comprise a comparison between space-based and terrestrial-based observations, an examination of the new fields opened by space-based telescopes, impact of space observations on science generally, a proposal for a specific experiment - no subject is barred. Length required is 2500 words maximum. One winner will be chosen for each Member State who will be invited to ESO H.Q. in Garching and three to five outstanding authors will be selected to visit the USA, or the Ariane launch centre in Kourou. Unfortunately closing date is 28 February so a rapid reaction is needed.

For further information ring ESA at ++ (33) (1) 42737155.

\section{Foreseeable Developments}

The number of scientific and technical applications being proposed for tethered satellites is continuously increasing. Generally speaking, unique capabilities of tethers are:

a) ability to deploy and retrieve over large distances masses connected to each other and hence practically unlimited capability for space transportation to or from space stations or other orbiting objects;

b)ability to produce a local controlled low gravity field, centred about an intermediate point between the two masses where the gravity is exactly zero;

c) possibility for a conducting tether to interact with the magnetic field of the Earth or other celestial bodies like the Sun, planets, etc., to make possible plasma research in unprecedented environments and even to produce energy;

d) possibility of momentum exchange between the two masses, by deploying or retrieving connected bodies.

Some typical examples of possible applications (selected from ref. [6]) are summarized in Figs. 6 and 7.

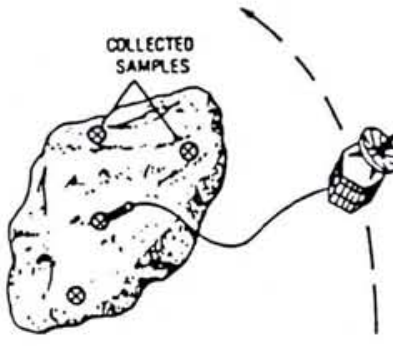

\section{RERERENCES}

(in chronological order)

Only a few individual papers are indicated. Rather, reference is made to recent general literature, in which other references can be found.

[1] Colombo G., Gaposchkin E.M., Grossi M.D., Smithson Astroph. Observ. Reports on Geoastronomy No. 1, Sept. 1974.

[2] The Tethered Satellite System, Final Report from the Facility Requirements Definition Team, May 1980 (NASA) 1980.

[3] Proc. Workshop on Applications of Tethers in Space, Williamsburg, Va. June $15-$ 17, 1983, Eds. P. Banks and C. Buongiorno (NASA) 1983

[4] Proc. Workshop on Applications of Tethers in Space, Venice October 15-17, 1985 Ed. W.A. Baracat (NASA CP 2422) 1986.

[5] Baracat W.A. and Butler C.L., Tethers in Space Handbook (NASA) 1986.

[6] Arnold D.A., op. cit. 35-50

[7] Tethers in Space, Proc. Int. Conf. Arlington, Sept. 17-19, Eds. P.M. et al., Advances in the Astronautical Sciences, 62 (1987).

[8] Laue J.H., Conference on Space Tethers for Science in the Space Station Era, Venice, October 1987.

[9] Proc. Tether Workshop, DFVLR KolnPorz, February 10, 1988, Ed. W. Seboldt.
Fig. 7-Sample collection from an asteroid for example [7]. 